\title{
A Neural Network Model for Detection Systems Based on Data Mining and False Errors
}

\author{
Se-Yul Lee ${ }^{1}$, Bong-Hwan Lee ${ }^{2}$, Yeong-Deok Kim ${ }^{3}$, Dong-Myung Shin ${ }^{4}$, \\ and Chan-Hyun Youn ${ }^{5}$ \\ ${ }^{1}$ Department of Computer Science, Chungwoon University,
}

San 29 Namjang-Ri, Hongseong-Eup, Hongseong-Gun, Chungnam, 350-701, Korea Piratedcwunet.ac.kr

${ }^{2}$ Department of Electrical \& Computer Engineering, University of Florida

Gainesville, FL 32611-6200, U.S.A.

bhlee@acis.ufl.edu

${ }^{3}$ Department of Computer Information Science \& Engineering, Woosong University,

17-2 Jayang-Dong, Dong-Gu, Daejeon, 300-718, Korea

ydkim@wsu.ac.kr

${ }^{4}$ IT Infrastructure Protection Division Applied Security Technology Team, Korea Information

Security Agency,

78 Karak-Dong, Songpa-Gu, Seoul, 138-160, Korea

dmshinakisa.or.kr

${ }^{5}$ School of Engineering, ICU

119 Munjiro, Yuseung-Gu, Daejeon, 305-732, Korea

chyounaicu.ac.kr

\begin{abstract}
Nowadays, computer network systems play an increasingly important role in our society. They have become the target of a wide array of malicious attacks that can turn into actual intrusions. This is the reason why computer security has become an essential concern for network administrators. Intrusions can wreak havoc on LANs. And the time and cost to repair the damage can grow to extreme proportions. Instead of using passive measures to fix and patch security holes, it is more effective to adopt proactive measures against intrusions. Recently, several IDS have been proposed and they are based on various technologies. However, these techniques, which have been used in many systems, are useful only for detecting the existing patterns of intrusion. It can not detect new patterns of intrusion. Therefore, it is necessary to develop a new technology of IDS that can find new patterns of intrusion. This paper investigates the asymmetric costs of false errors to enhance the detection systems performance. The proposed method utilizes a network model considering the cost ratio of false errors. Compared with false positive, this scheme accomplishes both security and system performance objectives. The results of our empirical experiment show that the network model provides high accuracy in detection. In addition, the simulation results show that effectiveness of probe detection can be enhanced by considering the costs of false errors.
\end{abstract}

\section{Introduction}

The rapid growth of network in information systems has resulted in the continuous research of security issues. One of key research areas is detection system that many companies have adopted to protect their information assets for several years. In order 
to address the security problems, many automated detection systems have been developed. However, between 2002- 2005, more than 200 new attack techniques were created and announced which exploited Microsoft's Internet Information Server (IIS), one of the most widely used Web servers. Recently, several detection systems have been proposed based on various technologies. A "false positive error" is an error that detection system sensor misinterprets one or more normal packets or activities as an attack. Detection system operators spend too much time distinguishing events. On the other hand, a "false negative error" is an error resulting from attacker is misclassified as a normal user.

It is quite difficult to distinguish intruders from normal users. It is also hard to predict all possible false negative errors and false positive errors due to the enormous varieties and complexities of today's networks. Thus, detection system operators rely on their experience to identify and resolve unexpected false error issues.

This study proposes a method to analyze and reduce the total costs based on the asymmetric costs of errors in the detection system. This study adopts the network model that has shown successful results for detecting and identifying unauthorized or abnormal activities from the networks [1]. The objective of the proposed method is to minimize the loss for an organization under an open network environment. This study employs the network model for detection. Furthermore, the study analyzes the cost effectiveness of the false error levels and presents experimental results for the validation of our detection model.

The remainder of this paper consists of four sections. The next section presents the introduction of detection systems and the studies of data mining approaches for detection systems. The research model of this study is addressed in detail in Section 3 . In Section 4, the asymmetric costs of false negative errors and false positive errors are validated by experimental results. Finally, this paper is concluded with the summary, contributions and limitations.

\section{Detection Systems}

An intrusion is an unauthorized access or usage of the resources of a computer system [2]. Intrusion Detection System (IDS) is software with functions of detecting, identifying, and responding to unauthorized or abnormal activities on a target system $[3,4]$. The goal of the IDS is to provide a mechanism for the detection of security violations either in real-time or batch-mode $[5,6]$. Violations are initiated either by outsiders attempting to break into a system, or by insiders attempting to misuse their privileges [7]. IDS collect information from a variety of systems and network sources, and then analyze the information for signs of intrusion and misuse [8].

The major functions performed by IDS are monitoring and analyzing user and system activity, assessing the integrity of critical system and data files, recognizing activity patterns reflecting known attacks, responding automatically to detected activity, and reporting the outcome of the detection process.

Intrusion detection can be broadly divided into two categories based on the detection method: misuse detection and anomaly detection. Misuse detection works by searching for the traces or patterns of well-known port attacks. Clearly, only known attacks that leave characteristic traces can be detected this way. This model of the 
normal user or system behavior is commonly known as the user of system profile. A major strength of anomaly detection is its ability to detect previously unknown attacks.

IDS are categorized according to the kind of audit source location they analyze. Most IDS are classified as either network based intrusion detection or a host based intrusion detection approach for recognizing and deflecting attacks. When IDS look for these patterns in the network traffic, they are classified as network based intrusion detection. When IDS look for attack signatures in the log files, they are classified as host based intrusion detection. In either case, these products look for attack signatures and specific patterns that usually indicate malicious or suspicious intent. Host based IDS analyze host bound audit sources such as operating system audit trails, system logs, and application logs. Network based IDS analyze network packets that are captures on a network.

The current IDS have contributed to identifying attacks using historical patterns. But they have difficulty in identifying attacks using a new pattern or with no pattern [9]. Previous studies have utilized a rule based approach such as USTAT, NADIR, and W\&S [10-12]. They lack flexibility in the rule to audit record representation. Slight variations in an attack sequence can affect the activity rule comparison to a degree that intrusion is not detected by the intrusion detection mechanism.

While increasing the level of abstraction of the rule base does provide a partial solution, it also reduces the granularity of the intrusion detection device. These limitations in rule based systems can be summarized as follows: the lack of flexibility and maintainability in the acquisition process of rules, lack predictive capability, lack of automatic learning capability, a high rate of false alarms or missing alarms, and difficulty in applying organizational security policies.

Many recent approaches to IDS have utilized data mining techniques. Known examples are the Computer Misuse Detection System (CMDS), the Intrusion Detection Expert System (IDES), and the Multics Intrusion Detection and Alerting system (MIDAS) using neural networks. These approaches build detection models by applying data mining techniques to large data sets of an audit trail collected by a system [13].

Data mining based IDS collect data from sensors which monitor some aspect of a system. Sensors may monitor network activity, system calls used by user processes, and file system accesses. They extract predictive features from the raw data stream being for detection. Data gathered by sensors are evaluated by a detector using a detection technique. Table 1 shows the studies of data mining applications for IDS.

Table 1. List of data mining applications in IDS [4-10]

\begin{tabular}{c|c}
\hline Detection method & Data mining methods \\
\hline \multirow{3}{*}{ Misuse } & CBR of Esmaili \\
& NN of Endler \\
& NN of Cannady \\
& GA of Balajinath \\
\hline \multirow{2}{*}{ Anomaly } & NN of Kumar \\
& NN of Endler \\
& NN of Bonifacio \\
& GA of Sinclair \\
\hline \multirow{2}{*}{ Network based } & CBR of Esmaili \\
& NN of Heatley \\
& GA of Balajinath \\
\hline
\end{tabular}




\section{Cost of Errors for Detection Systems}

\subsection{Network Models for Detection Systems}

The model consists of network based detection model and monitoring tool (Fig. 1) [14]. The model adopts the problem solving methodology which uses previous problem solving situations to solve new problems.

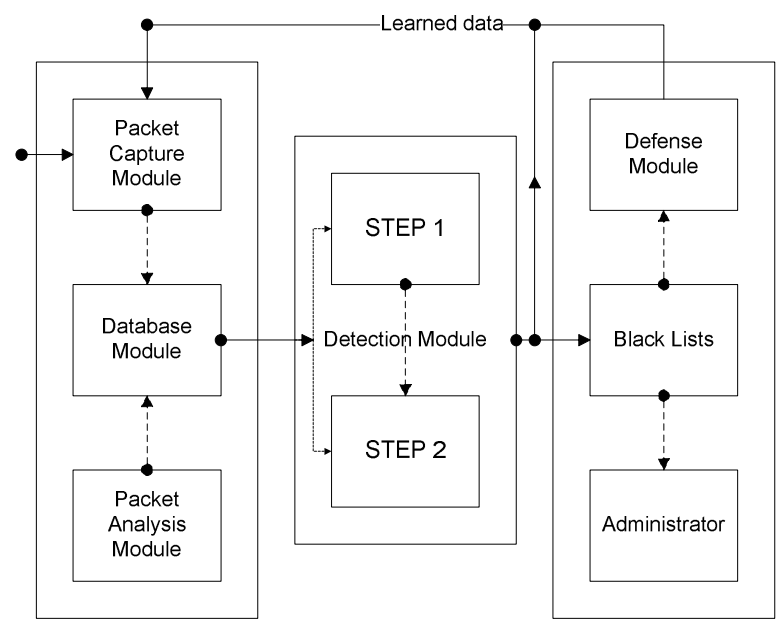

Fig. 1. Architecture of the proposed model

The model does preprocessing by packet analysis module and packet capture module. The packet capture module captures and controls packet. The packet capture module does real-time capturing and packet filtering by using the monitoring tool of Detector4win version 1.2 [15]. In the packet filtering process, packets are stored according to the features which distinguish between normal packets and abnormal packets. The packet analysis module stores data and analyzes half-open state. After storing packets, the packets, which are extracted by audit record rules in the packet analysis module, are sent to the detection module.

The input and output of detection module, namely STEP 1 [16], is traffic and alert, respectively. The traffic is an audit packet and the alert is generated when an intrusion is detected. The detection module consists of session classifier, pattern extractor, and pattern comparator. The session classifier takes packet of the traffic and checks whether or not the source is the same as the destination. There is a buffer for the specific session to be stored. And, if the next packet is arrived, it is stored in the corresponding buffer. If all packets of the corresponding buffer are collected, all packets of the corresponding buffer are output as on session. The output session becomes an input to the pattern extractor or pattern comparator according to action mode. The action mode consists of learning mode and pre-detection mode. The output session from the session classifier is sent to the pattern extractor in the learning mode and to the pattern comparator in the pre-detection mode. Fig. 2 shows the block 
diagram of the STEP 1. The pattern extractor collects the sessions, which have the same destination, and extract common pattern. Each consists of two features. The first feature is a head part which appears in common sessions, which have the same destination, when sessions are arranges by size packets using the time sequence. The second feature is the minimum length of the sessions which have the same destination. The length of session is the number of packets of session.

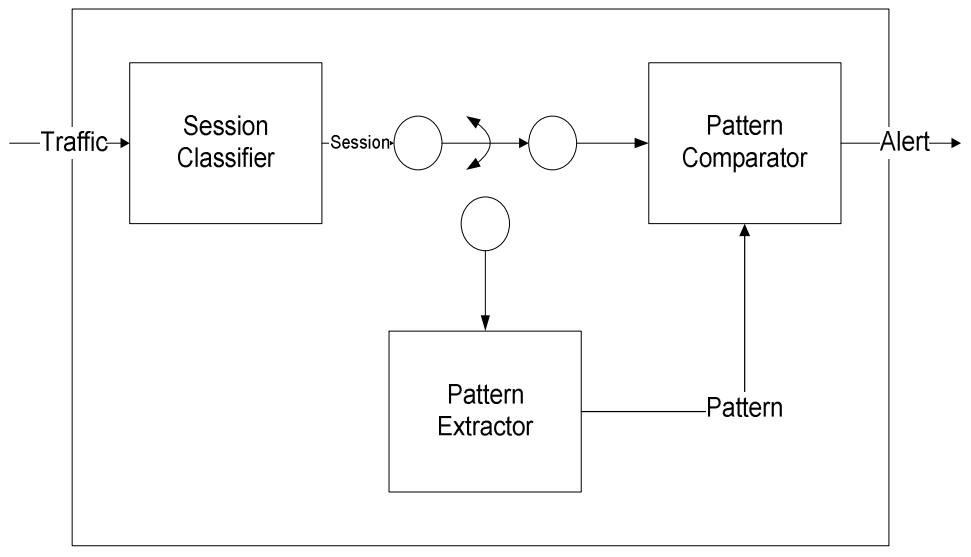

Fig. 2. A Block Diagram of STEP 1

The pattern comparator compares packets with the rule based pattern. If the probe packets and the rule based pattern do not correspond, the pattern comparator considers the probe packets as the abnormal session and generates an alert signal. Thus, the pattern comparator receives a session and the rule based pattern as an input. From the input session the data size and the length of session are extracted. If there is a mismatch in one of two features, the pattern comparator considers a session as the abnormal session. What we must consider for the pattern extraction is whether we extract the pattern continuously or we extract the pattern periodically. We generally call the former the real-time pattern extraction and the latter the off-line pattern extraction. The real-time patterns extraction is better than off-line pattern extraction in the viewpoint of updating the recently changed pattern. But, it is difficult to update the pattern when probes occur. For the pattern, if possible, normal traffic becomes a rule-based pattern. Otherwise, an abnormal traffic sometimes becomes a rule-based pattern. And an abnormal intrusion traffic is considered as an normal traffic. It is called false negative error. The model uses detection module, namely STEP 2, to compensate the false negative error by using fuzzy cognitive maps.

The detection module of model is intelligent and uses causal knowledge reason utilizing variable events which hold mutual dependences. For example, because CPU capacity increases when syn packet increases, the weight of a node, $W_{i k}$, has the value of rang from 0 to 1 . The total weighted value of a node depends on path between nodes and iteration number. This can be written as the following equation. 


$$
N_{k}\left(t_{n+1}\right)=\sum_{i=1}^{n} W_{i k}\left(t_{n}\right) N_{i}\left(t_{n}\right),
$$

where

$N_{k}\left(t_{n}\right)$ : Tthe value of the node $k$ at the iteration number $t_{n}$

$t_{n}:$ Iteration number

$W_{i k}\left(t_{n}\right)$ : Weight between the node $i$ and the node $k$ at the iteration number $t_{n}$

On the above equation, the sign of weight between the node $i$ and the node $k$ depends on the effect from the source node to the destination node.

\subsection{Analysis for Costs of Errors}

For the network modeling in the Fig. 1, the analysis of costs of errors is presented in Fig. 3. The purpose of Fig. 3 is to analyze the relationship between the total costs and detection system errors, and find the optimal threshold of network model that minimizes the total costs for intrusion detection.

The solution provides the weights of errors while the weights can be adjusted to enhance the effectiveness of intrusion detection according to the threshold value of the activation function. The activation function produces the level of excitation by comparing the sum of these weighted inputs with the threshold value. This value is entered into the activation function, i.e. the sigmoid function, to derive the output from the node.

The cost of attacks or errors has received attention in designing IDS [17], [18]. The cost of a false negative error is much higher than that of a false positive error because an organization may suffer from various security incidents compromising confidentiality,

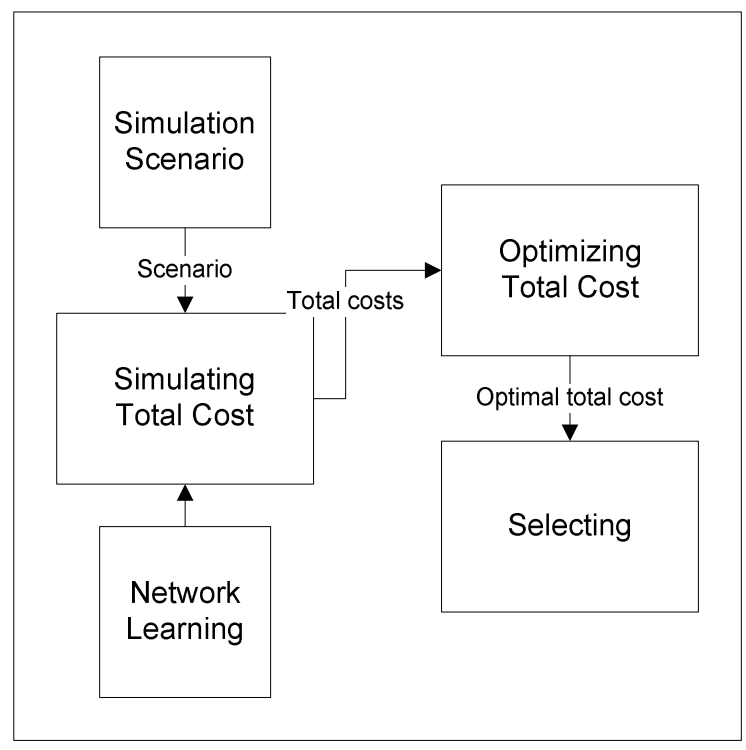

Fig. 3. A Block Diagram of Error's Cost 
integrity, and availability when not detecting real attacks. This study introduces the concept of the asymmetric costs of errors to calculate overall misclassification costs. The performance of detection system is optimized when the total costs are minimized.

A false negative error, which is the cost of not detecting an attack, is incurred when the detection system does not function properly and mistakenly ignores an attack. This means that the attack will succeed and the target resource will be damaged. Thus, a false negative error should take a higher weight than a false positive error. The false negative errors are therefore described as the damage cost of the attack. The cost function for detection system can be defined as follows:

$$
\begin{aligned}
A_{\text {total }}(x) & =\omega_{1} A_{1}+\omega_{2} A_{2}+\ldots . .+\omega_{n} A_{n} \\
& =\sum_{i=1}^{n} \omega_{i} A_{i},
\end{aligned}
$$

where

$$
\begin{aligned}
& A_{\text {total }}(x): \text { Total cost } \\
& \omega_{i}: \text { Weight for each cost } A_{i} \\
& A_{i}: \text { Cost for each error } i
\end{aligned}
$$

To measure each cost, we used the errors that are the misclassified by our detection methods. The cost ratio of a false positive error and a false negative error varies depending on the characteristics of the organization. Thus, we found out the minimal total costs by the simulation of adjusting the weights one hundred times. The threshold values can be searched to minimize the total costs for a specific cost ratio of false negative errors to false positive errors.

\section{Performance Evaluation}

For the performance evaluation of the proposed model, we have used the KDD data set (Knowledge Discovery Contest Data) by MIT Lincoln Lab, which consists of labeled data (training data having syn and normal data) and non-labeled data (test data). We utilize a network model to apply the proposed method for the above data. Three-layer feed-forward networks are used to detect an intrusion. Logistic activation function is utilized in the output layer. The number of hidden nodes is selected through experimentation with $\mathrm{n} / 2, \mathrm{n}$, and $2 \mathrm{n}$ of nodes ( $\mathrm{n}$ is the sum of input nodes) by fixing the input and output nodes. A series of experiments were conducted to analyze the effects of varying the value of the threshold values of false negative errors and false positive errors (Fig. 4).

As the threshold value increases, false positive errors increase while false negative errors decrease. After the ratio of false negative errors over false positive errors is given, the threshold value that minimizes the total cost can be determined. Let us suppose that the cost of false negative error is equal to that of false positive error. We can find the optimal point of the threshold at 0.12 from Fig. 4 . When the output is over the threshold value, the output is interpreted as an attack and normal vice versa. The performance of networks is calculated by the function of cost, which consists of false positive errors and false negative errors (Table 2). 


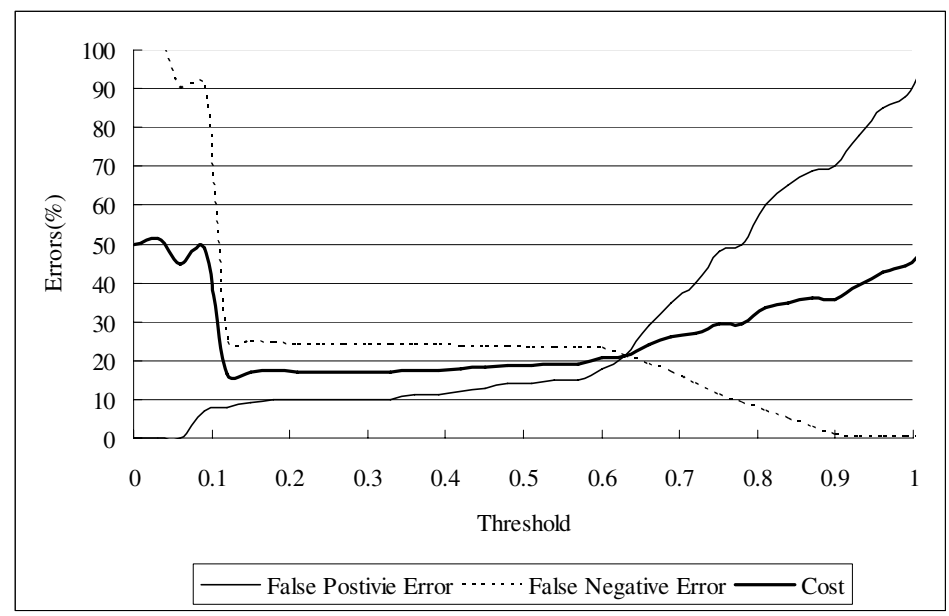

Fig. 4. The performance of the proposed model with cost of errors

The performance of network model is measured in the output sample data. The total cost of the network model is $15.32 \%$ when the threshold value is 0.5 which is a general value without considering costs of errors. When the optimal point of threshold of 0.12 is applied to the network model from Fig. 4, the cost is $15.95 \%$. The cost decreases and the performance of the intrusion detection model are sensitive according to the threshold.

A false negative error is more important in detection system as mentioned in the previous section. We need to concentrate on the decrease of false negative errors according to the change of the threshold value. The decreases of the false negative errors are $1.17 \%$ from 9.01 to $7.84 \%$. The change in the total cost would be greater as weights are added to the negative false errors.

Thus, we will analyze the results of the network model by the simulation for total costs of detection systems performance. We increase the cost ratio by 0.1 from 1.0 and 10.0 and search each minimal total by 250 times through the simulation.

When a false negative error takes the weight value five times larger than a false positive error, the total percentage of errors is $9.95 \%$. When the cost ratio is 1 , the total percentage of errors is $15.94 \%$. The decreased amount is about $38 \%$ compared to

Table 2. The performance of network models

\begin{tabular}{c|c|c|c|c}
\hline $\begin{array}{c}\text { Threshold } \\
\text { Value }\end{array}$ & Sample & $\begin{array}{c}\text { False positive } \\
\text { errors(\%) }\end{array}$ & False negative errors(\%) & Cost(\%) \\
\hline \multirow{3}{*}{0.5} & Input & 24.63 & 9.23 & 16.93 \\
\cline { 2 - 5 } & Output & 21.63 & 9.01 & 15.32 \\
\cline { 2 - 5 } & Total & 23.13 & 9.12 & 16.13 \\
\hline \multirow{3}{*}{0.12} & Input & 25.49 & 8.45 & 16.97 \\
\cline { 2 - 5 } & Output & 24.06 & 7.84 & 15.95 \\
\cline { 2 - 5 } & Total & 24.78 & 8.15 & 16.46 \\
\hline
\end{tabular}


the original cost, which has the cost ratio of 1 . Thus we come to the conclusion that a success factor for detection system is the cost ratio and threshold as well as the classification accuracy.

\section{Conclusions}

There have been a variety of studies and systems designed to detect intrusion by using data mining approaches. However, most studies addressed the measure of system performance as providing prediction accuracy without considering the costs of errors in intrusion detection. In this study we proposed a network model based on costs of false positive errors and false negative errors. The first diagram of this study develops a network model for intrusion detection, while the second diagram analyzes the system performance based on costs of errors.

The results of the empirical experiment indicate that the network model provides very high performance in accuracy of intrusion detection. The cost of false negative errors must be much higher than that of the false positive errors to an organization. The total cost of errors is minimized by adjusting the threshold value for the specific cost ratio of false negative errors to false positive errors.

For further study, other data mining methods such as genetic algorithms and inductive learning may be applied to detection system.

\section{Acknowledgements}

This work was supported by University IT Research Center Project of MIC. It is also supported in part by MOCIE Regional Innovation Program.

\section{References}

1. Lee, W., Stolfo, S. J., "A data mining framework for building intrusion detection models," IEEE Symposium on Security and Privacy, pp. 209-220, 1999.

2. Es,ao;o, M., Safavi-Naini, R., Balachadran, B., "Case-based reasoning for intrusion detection," 12th Annual Computer Security Application Conference, pp. 214-223, 1996.

3. Denning, D. E., "An intrusion detection model," IEEE Trans. S. E., SE-13(2), pp. 222-232, 1987.

4. Richards, K., "Network based intrusion detection: a review of technologies," Computer and Security, pp. 671-682, 1999.

5. Debar, H., Dacier, M., "Towards a taxonomy of intrusion detection systems," Computer Networks, pp. 805-822, 1989.

6. Debar, H., Becker, M., "A neural network component for an intrusion detection system," IEEE Computer Society Symposium Research in Security and Privacy, pp. 240-250, 1992.

7. Weber, R., "Information Systems Control and Audit," IEEE Symposium on Security and Privacy, pp. 120-128, 1999.

8. Lippmann, R. P., "Improving intrusion detection performance using keyword selection and neural networks," Computer Networks, Vol. 24, pp. 597-603, 2000. 
9. Jasper, R. J., Huang, M. Y., "A large scale distributed intrusion detection framework based on attack strategy analysis," Computer Networks, Vol. 31, pp. 2465-2475, 1999.

10. Ilgun, K., Kemmerer, R. A., "Ustat: a real time intrusion system for UNIX," Proceedings of the IEEE Symposium on Research in Security and Privacy, pp. 16-28, 1993.

11. Hubbards, B., Haley, T., McAuliffe, L., Schaefer, L., Kelem, N., Walcott, D., Feiertag, R., Schaefer, M., "Computer system intrusion detection," pp. 120-128, 1990.

12. Vaccaro, H. S., "Detection of anomalous computer session activity," Proceedings of the IEEE Symposium on Research in Security and Privacy, pp. 280-289, 1989.

13. Helman, P., "Statistical foundations of audit trail analysis for the detection of computer misuse," IEEE Transactions on software engineering, Vol. 19, pp. 861-901, 1993.

14. Lee, S. Y., "Design and analysis of probe detection systems for TCP networks," International Journal of Advanced Computational Intelligence \& Intelligent Informatics, Vol. 8, pp. 369-372, 2004.

15. Lee, S. Y., An Adaptive probe detection model using fuzzy cognitive maps, Ph. D. Dissertation, Daejeon University, 2003.

16. Park, S, J., A Probe Detection Model using the analysis of the Session Patterns on the Internet Service, ph. D. Dissertation, Daejeon University, 2003.

17. Maxion, R. A., "Masquerade detection truncated command lines," International Conference on Dependable Systems and Networks, pp. 219-228, 2002.

18. Joo, D, J., The Design and Analysis of Intrusion Detection Systems using Data Mining, Ph. D. Dissertation, Korea Advanced Institute of Science and Technology, 2003. 